\title{
Herpes simplex virus tipo 1 como factor de riesgo asociado con la enfermedad de Alzheimer
}

\author{
CAROLINA MARTIN ${ }^{1, \mathrm{a}}$, LORETTO SOLÍS ${ }^{1, \mathrm{~b}}$, \\ MARGARITA I. CONCHA ${ }^{2, c}$, CAROLA OTTH ${ }^{1, c}$
}

\section{Herpes simplex virus type 1 as risk factor associated to Alzheimer disease}

Herpes simplex virus type 1 (HSV-1) is ubiquitous, neurotropic, and the most common pathogenic cause of sporadic acute encephalitis in humans. Herpes simplex encephalitis is associated with a high mortality rate and significant neurological, neuropsychological, and neurobehavioral sequels. HSV-1 infects limbic system structures in the central nervous system (CNS), and has been suggested as an environmental risk factor for Alzheimer's disease. The possibility that HSV-1 reactivates in CNS neurons causing chronic progressive damage at cellular level and altering the neuronal functionality has not been thoroughly investigated. Currently it is ignored if recurrent reactivation of HSV-1 in asymptomatic patients involves some risk of progressive deterioration of the CNS functions caused, in example, by a neuroinflammatory response against the virus or by direct toxicity of the pathogen on neurons. Therefore, studies regarding the routes of dissemination of $H S V-1$ from the peripheral ganglions to the CNS, as well as the possible cellular and molecular mechanisms implied in generating neuronal damage during latent and productive infection, are of much relevance.

(Rev Med Chile 2011; 139: 779-786).

Key words: Alzheimer disease; Encephalitis, viral; Herpesvirus 1, human.

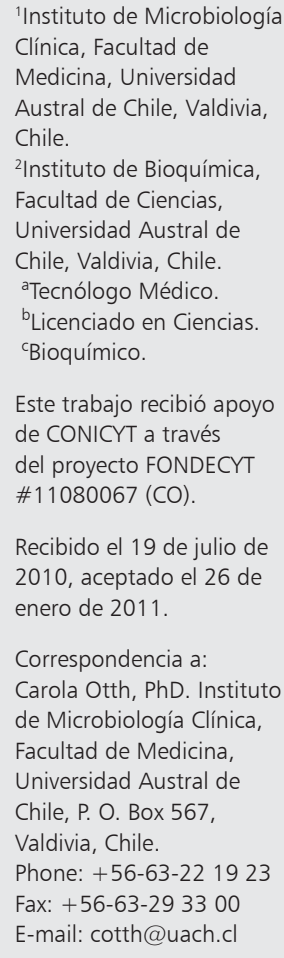

\section{Herpes simplex virus tipo 1}

L

a familia Herpesviridae, se encuentra ampliamente diseminada en la naturaleza. Existen más de 100 tipos diferentes de herpesvirus, los que presentan la misma estructura, ADN lineal de doble hebra dentro de una cápside icosaédrica, rodeada por un tegumento proteico y cubierto por una envoltura lipídica (Figura 1) $)^{1,2}$. Una característica que distingue a los herpesvirus, es su capacidad para establecer infecciones persistentes latentes en el hospedero infectado, estado en el cual el genoma viral se encuentra en forma episomal en el núcleo de la célula infectada, con una expresión limitada de genes específicos para el mantenimiento del estado de latencia (transcritos
LAT) y sin producir partículas virales infectivas ${ }^{3,4}$ Este tipo de infección se piensa que constituye una estrategia viral para evadir su detección por parte del sistema inmune. Bajo ciertas condiciones -incluyendo la exposición a radiación UV, estrés emocional, alteración del balance hormonal, depresión del sistema inmunológico, entre otros- se puede interrumpir el estado de latencia, induciendo una reactivación del genoma viral, con la consecuente producción de nuevas partículas virales infectivas que inician la recurrencia del cuadro clínico ${ }^{1,4}$. Sin embargo, los mecanismos celulares y moleculares de este proceso, aún se desconocen en detalle.

En base al tipo de célula en que establecen latencia y otras características, los herpesvirus humanos han sido clasificados en distintas 


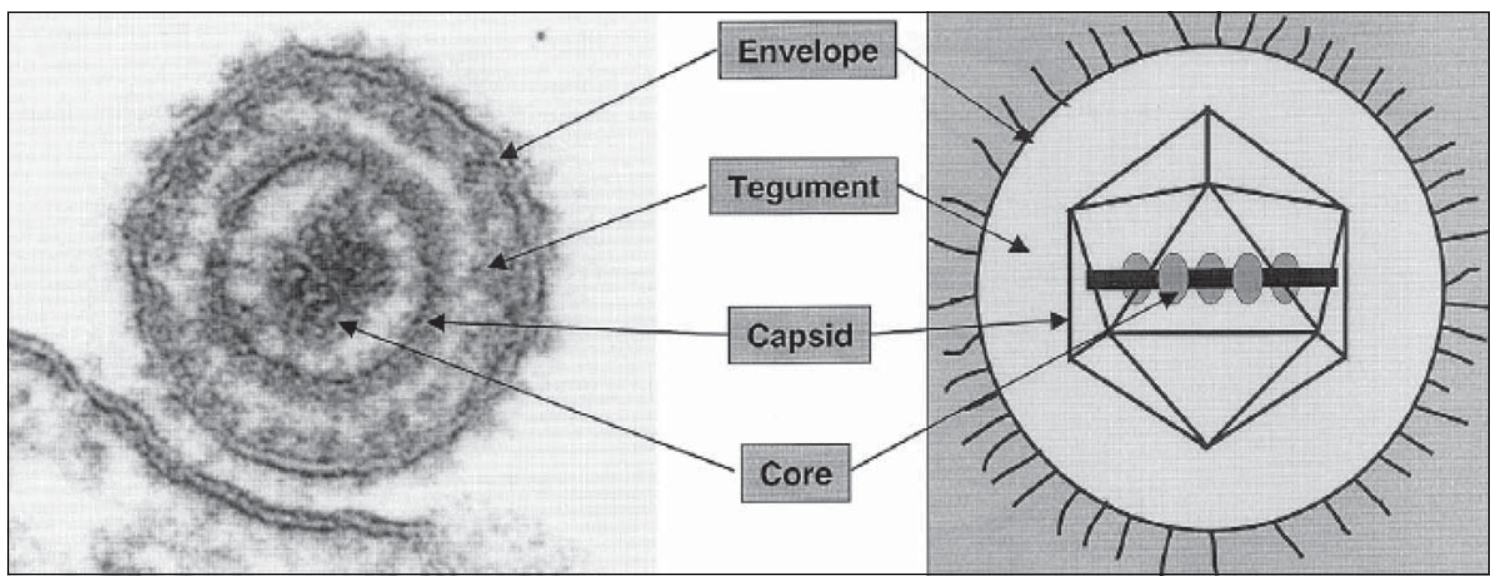

Figura 1. Morfología del virión de los Herpesvirus. Es posible diferenciar cuatro estructuras: el core, que contiene el DNA lineal de doble hebra, una cápside icosaédrica rodeada por un tegumento proteico y la envoltura lipídica que contiene -insertas- proteínas glicosiladas. (Mettenleiter y col., 2003 . Copyright (C) 2010 Elsevier B.V.).

subfamilias. Herpes simplex virus tipo 1 (HSV1) pertenece a la subfamilia Alfaherpesvirinae, cuyos miembros se caracterizan por presentar un mecanismo de daño lítico y ser neurotrópicos. HSV-1 es un patógeno humano ubicuo que infecta aproximadamente $90 \%$ de la población adulta ${ }^{5,6}$. El sitio inicial de infección es la mucosa oral, epitelio corneal o heridas en la piel, en donde la replicación viral causa lesiones vesiculares. El estado de latencia es establecido en neuronas sensoriales que se encuentran inervando el sitio de infección inicial ${ }^{4,5,7}$. HSV-1 alcanza los terminales de nervios periféricos, donde la nucleocápside viaja por transporte axonal retrógrado hacia los cuerpos neuronales alcanzado el núcleo. El proceso de reactivación conlleva al transporte axonal anterógrado de la nueva progenie viral hacia el sitio inicial de infección, conduciendo a una potencial manifestación recurrente de los síntomas clínicos y a la posibilidad de transmisión ${ }^{1,8}$.

\section{Infección del sistema nervioso central por HSV-1}

Las primeras evidencias de que HSV-1 podía ser transportado hasta el sistema nervioso central (SNC) desde el sistema nervioso periférico, fueron obtenidas a partir de estudios acerca de la encefalitis causada por herpes simplex (HSE) 5,9-12. $^{2}$.

Los terminales de nervios sensoriales perifé- ricos que se encuentran inervando el sitio inicial de infección, son invadidos rápidamente y el virus es transportado a través de los axones hacia el ganglio periférico, donde establece una infección persistente latente.

En algunas circunstancias, el virus continúa su diseminación centrípetamente, alcanzando el cerebro y causando encefalitis ${ }^{5,8,11}$. HSV es la causa más común de encefalitis aguda no epidémica, con una frecuencia anual estimada de un caso por 250.000 a 500.000 habitantes ${ }^{5,6,11,12}$. La mortalidad asociada a HSE es superior a 70\% en ausencia de un tratamiento antiviral efectivo y los sobrevivientes presentan secuelas neurológicas relacionadas con alteraciones cognitivas, memoria y personalidad ${ }^{5,11}$. Aciclovir es el tratamiento de elección en caso de HSE pero, aun bajo medicación, la mortalidad continúa siendo alta. Sobre la base de estudios serológicos, se ha establecido que cerca de un tercio de los casos de HSE son causados por infección primaria con HSV-1, mientras que dos tercios resultan de la reactivación viral ${ }^{11,12}$.

El daño inflamatorio y el edema que se produce durante HSE ocurre en el lóbulo frontal y el lóbulo temporal incluyendo el sistema límbico ${ }^{10,13}$. Al respecto, se ha encontrado la presencia de antígenos virales de HSV en cerebros de pacientes fallecidos por HSE concentrados principalmente en los lóbulos temporal inferior y medio, hipocampo, amígdala, corteza olfatoria, ínsula y giro cingulado $^{14,15}$. La localización del virus en HSE podría 


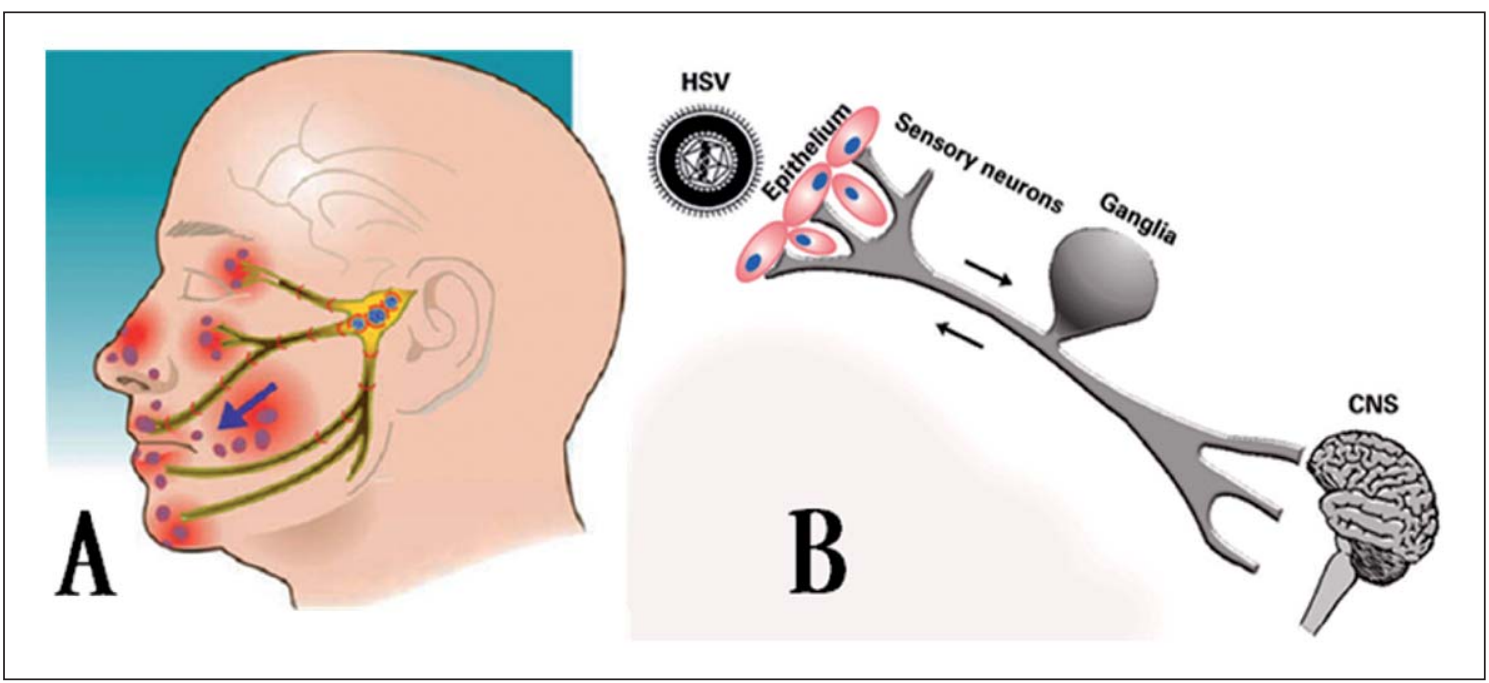

Figura 2. Diagrama esquemático del ciclo de vida de HSV-1. A) Se observan las posibles vías de entrada del HSV- 1 y posterior reactivación viral y recurrencia clínica. B) HSV viaja por transporte axonal retrogrado hacia el ganglio sensorial donde establece latencia. En ciertas condiciones, HSV alcanza el SNC, probablemente vía neuronal (Adaptado de Perkins, 2002 ${ }^{8}$. Copyright (C) 2000-2010 by John Wiley \& Sons, Inc.).

ser explicada debido a la ruta por la cual el virus alcanza el cerebro. En particular, dos vías han sido propuestas, las que involucran el flujo intraneuronal del virus a lo largo del nervio olfatorio o a través de ramas del nervio trigémino que inervan las meninges basales. El acceso del virus por la ruta olfatoria puede explicar mejor la distribución de lesiones en el sistema límbico, incluyendo las vías olfatorias (Figura 2) ) $^{8,15}$.

Estudios sobre los mecanismos moleculares de infección neuronal de los herpesvirus, han demostrado la particular capacidad de diseminación direccional trans-neuronal de HSV, a través de las sinapsis. De hecho, existe evidencia de la participación de varias glicoproteínas de la envoltura viral, tales como $\mathrm{gD}, \mathrm{gB}$ y gE, en la propagación trans-sináptica de los virus. De este modo el virus puede acceder a circuitos neuronales ligados sinápticamente ${ }^{1}$.

Por otra parte, existen estudios que demuestran que la vulnerabilidad a una infección viral, además de estar ligada a un deterioro inmunológico inherente a la senectud, depende de una potencial sensibilidad genética. Lee y col $(2001)^{16}$ utilizaron un modelo de infección experimental evaluando distintas cepas de ratón y demostraron que la susceptibilidad de los ratones BXD-8, a la infección por citomegalovirus - un virus perteneciente a la familia Herpesviridae-capaz de invadir y producir un daño considerable en el cerebro, está asociada con una deleción en el gen Klra8, el cual se relaciona con la regulación de las células natural killer. En cambio, los ratones C57BL/6 se mostraron resistentes a la infección. Interesantemente, Thach y col $(2000)^{17}$ observaron al infectar ratones con el virus Sindbis Neuroadaptado (NSV), que $100 \%$ de los ratones C57BL/6 sufrió encefalitis fatal, mientras que todos los ejemplares de la cepa BALB/cBy sobrevivieron a la infección. Ambas investigaciones evidencian la importancia de la relación entre el background genético y la infección con virus específicos. Adicionalmente, un estudio utilizando cultivo de oligodendrocitos humanos de diferentes donantes demostró que también existe una resistencia diferencial a la infección con HSV-1 dependiendo del donante, sugiriendo que en seres humanos esta resistencia a la infección también estaría determinada genéticamente ${ }^{18}$.

\section{HSV-1 como factor de riesgo asociado con la enfermedad de Alzheimer}

Durante el envejecimiento fisiológico es posible observar una serie de alteraciones que conllevan a una deficiencia de la actividad cerebral, la 
cual es más evidente en los casos de enfermedades neurodegenerativas, en donde circuitos nerviosos responsables del mantenimiento de los procesos cerebrales cognitivos se ven afectados ${ }^{19}$. Debido a la normal alteración de las funciones del sistema inmunológico - producto del envejecimiento-, la vulnerabilidad del SNC a la invasión por diferentes tipos de agentes infecciosos se ve aumentada con la edad ${ }^{20,21}$.

En el largo plazo, episodios de reactivaciones clínicas y sub-clínicas a nivel del SNC, que generen un proceso inflamatorio crónico por parte de la glía, podrían tener una participación (hasta el momento desconocida) sobre la integridad neuronal, con riesgo de producir disfunción neuronal e inducir eventos neurodegenerativos progresivos ${ }^{22,23}$.

Un hallazgo interesante fue el que estableció que las estructuras límbicas afectadas por HSE son las mismas afectadas en la enfermedad de Alzheimer (EA), lo cual condujo a la hipótesis propuesta en los primeros años de la década 1980-89, que planteaba que HSV-1 podría estar involucrado en la patogénesis de la $\mathrm{EA}^{24}$. Aunque la HSE es una enfermedad muy distinta a la EA, a largo plazo conduce a ciertos efectos clínicos posteriores tales como pérdida de memoria y declive cognitivo, los cuales son característicos de la $\mathrm{EA}^{25}$. Varios agentes infecciosos, particularmente virus, han sido propuestos como potenciales factores de riesgo asociados a la aparición de EA. Esto último, basado en la capacidad de algunos virus, como HSV-1, para establecer latencia en el tejido neuronal, con la subsecuente posibilidad de producir daño celular progresivo debido a su reactivación.

La EA es una enfermedad neurodegenerativa crónica, caracterizada histológicamente por la acumulación intracelular de ovillos neurofibrilares (ONF) compuestos por la proteína tau, asociada a microtúbulos, la cual se encuentra anormalmente hiperfosforilada ${ }^{26}$. Además, ocurre la formación de depósitos extracelulares de péptido beta-amiloide $(\mathrm{A} \beta)$ y muerte neuronal masiva en los estadios avanzados de la enfermedad ${ }^{26-28}$. Los pacientes con EA manifiestan deterioro de sus capacidades cognitivas, memoria y alteraciones de la personalidad. La etiología de la EA es multifactorial, pero existe $5-10 \%$ de los casos en los cuales la enfermedad es hereditaria con una transmisión autosómica dominante ${ }^{26-28}$. El HSV-1 es uno de los candidatos propuestos como posible factor de riesgo en el desarrollo de EA. Esto debido a su carácter neurotrópico, ubicuo en la población general, capaz de establecer latencia en SNC y, además del declive inmunológico producto del envejecimiento, con lo cual podría aumentar la reactivación en los adultos generando un daño neuronal progresivo, posiblemente debido al proceso inflamatorio crónico localizado o quizás por acción directa del virus sobre la funcionalidad neuronal.

$\mathrm{Al}$ respecto, la neuroinflamación se encuentra asociada a la activación de las células gliales, las cuales inducen inicialmente una respuesta inmune a través de la activación de diferentes receptores de reconocimiento de patrones asociados a patógenos, incluyendo los receptores tipo Toll (TLRs), los cuales activan respuestas inflamatorias e inmunomoduladoras induciendo a su vez la expresión de genes antimicrobiales y citoquinas proinflamatorias. Algunos TLRs que se expresan en células microgliales son críticos en detectar y generar respuestas defensivas contra patógenos que infectan el SNC. Recientemente, se ha demostrado que esta inflamación excesiva que se genera durante la encefalitis herpética sería en gran parte producto de la activación de los TLRs, con la consecuente expresión de citoquinas inflamatorias $^{29,30}$. Un estudio que avala fuertemente este hallazgo es el realizado por Kurt-Jones y col $(2004)^{31}$, donde demuestran que TLR2 induce en ratones la generación de citoquinas inflamatorias en respuesta al HSV-1, mientras que en ratones carentes de TLR2 funcionales, se detecta una reducción en los síntomas de la encefalitis herpética. Además de ello, se ha reportado que ratones neonatos infectados con HSV-1 deficientes en TLR2, secretan menores cantidades de IL-6 y poseen una alta tasa de sobrevivencia comparada con los ratones control ${ }^{32,33}$. Todos estos antecedentes demuestran la contribución de los TLRs en activar eventos neuroinflamatorios por acción de patógenos neurotrópicos.

La hipótesis que relaciona a HSV-1 con la patogénesis de la EA ha adquirido relevancia debido a que se han detectado secuencias de $\mathrm{ADN}$ viral ${ }^{34,35,36}$ o antígenos virales y cuerpos de inclusión intranucleares en astrocitos ${ }^{37}$ obtenidos de cerebros de personas que padecieron EA. Adicionalmente, se ha demostrado que la presencia de HSV-1 en el cerebro de portadores del alelo $\varepsilon 4$ de la apoliproteína $\mathrm{E}$ (apoE) constituye un fuerte factor de riesgo para desarrollar la $\mathrm{EA}^{38-40}$. ApoE tiene una importante 
función en el transporte de lípidos en la sangre y en la redistribución de lípidos entre células en el cerebro. Además, el alelo $\varepsilon 4$ (a diferencia de los otros alelos) se asocia con una disminuida reparación del SNC después de lesiones ${ }^{41,42}$ y además se ha demostrado que apoE $\varepsilon 4$ favorece la invasión del herpesvirus desde la sangre al cerebro ${ }^{43}$. Asimismo, se ha encontrado que la frecuencia de reactivación viral es mayor en personas homocigotas para el alelo $\varepsilon 4$ del gen de la apoE ${ }^{34,36}$, sugiriendo que factores virales y genéticos pueden estar involucrados en EA. En investigaciones recientes, se ha encontrado la presencia de anticuerpos anti-HSV en líquido cefalorraquídeo de personas con EA y en personas mayores sin antecedentes de EA, no así en líquido cefalorraquídeo en niños menores de 7 años ${ }^{20}$. Al respecto, un reciente estudio poblacional prospectivo evidenció que existe un significativo riesgo de desarrollar EA en pacientes mayores que presentan anticuerpos IgM contra HSV-1, además de los factores de riesgo conocidos (edad, educación, apoE ${ }^{44}$. Este hallazgo se sustenta, además, en que durante una HSE, IgM anti-HSV-1 puede perdurar por 56 a 328 días y también en que se ha reportado una alta prevalencia de casos con tasas importantes de IgM durante reactivaciones por HSV- $1^{44}$. Estos antecedentes sugieren la existencia de reactivaciones recurrentes asociadas a la infección neuronal por HSV en personas adultas, debido posiblemente a un declive inmunológico y activación localizada del sistema inmune innato, generando un eventual riesgo de patologías neuronales producto de disfunción neuronal. Por otra parte, se ha demostrado que una porción de la glicoproteína B de HSV-1 tiene una alta homología con $A \beta$, la cual forma fibras análogas a las fibras de $A \beta$ con un efecto neurotóxico sobre cultivos neuronales ${ }^{45}$.

Los eventos de hiperfosforilación anormal de tau han sido descritos en diferentes patologías como taupatías, enfermedad de Alzheimer, e incluso en sinucleopatías como la enfermedad de Parkinson. La detección de estos eventos ha sido posible por la utilización de anticuerpos específicos que detectan epítopes fosforilados de tau, característicos de la EA. Así, AT8 detecta a Ser202 y Treo205 fosforiladas, Tau1 detecta los mismos epítopes pero desfosforilados y PHF-1 (filamentos helicoidales pareados) detecta las serinas 396 y 404 fosforiladas. Estos eventos de hiperfosforilación son previos a la formación de estructuras anómalas generadas por la autoagregación de las formas hiperfosforiladas de tau, que forman una red filamentosa y compacta. Se ha descrito la hiperfosforilación de tau por acción de diversos estímulos neurotóxicos que, además, inducen apoptosis, como por ejemplo lo que ocurre en animales sometidos a isquemia transciente ${ }^{46}$ o por alteración del metabolismo de la glucosa ${ }^{47}$; en cultivos neuronales tratados con radicales libres ${ }^{48}$, en neuroblastomas tratados con plaquitaxel y doxorubicina (compuestos anticancerígenos ${ }^{49}$, en neuroblastomas sometidos a estrés hiperosmótico ${ }^{50} \mathrm{e}$ incluso tratados con inhibidores de la quinasa de supervivencia PI3K $\mathrm{K}^{51}$. Todos estos hallazgos indican que los eventos de hiperfosforilación de tau, ocurren en la vía de activación del proceso apoptótico, quizás como requerimiento para todos los cambios que ocurren a nivel celular que finalizan con la generación de los cuerpos apoptóticos. Además, sugieren que los procesos apoptóticos y la neurodegeneración del citoesqueleto se encuentran estrechamente asociados, y ocurren por una diversidad de estímulos neurotóxicos ${ }^{49}$. Al respecto recientemente hemos demostrado que cultivos neuronales y gliales infectados con HSV-1 sufren un deterioro de sus procesos dendríticos y alteración de la dinámica microtubular, indispensables para los procesos neuronales ${ }^{52}$. Además de ello, encontramos que HSV-1 induce hiperfosforilación de epítopes de tau de tipo Alzheimer, presentando una estrecha analogía a los procesos de hiperfosforilación descritos en enfermedades neurodegenerativas ${ }^{52,53}$. Estos hallazgos fueron luego corroborados por el grupo de Itzhaki ${ }^{54}$. Es más, recientemente hemos demostrado que además de ser hiperfosforilada tau sufre procesamiento proteolítico por acción de caspasa-3 activa durante infección de neuronas y astrocitos con HSV- $1^{55}$, evento descritos que aceleran la autoagregación anómala de tau produciendo un desmantelamiento de los microtubulos, alteración de componentes del citoesqueleto y deterioro del funcionamiento neuronal.

Todos estos hallazgos, y considerando que a nivel del SNC HSV-1 puede establecer latencia en áreas frontal, temporal e hipocampo ${ }^{56,57}$, sugieren que HSV-1 podría estar implicado en la inducción de eventos neuropatológicos reiterados a lo largo de la vida, pudiendo constituir un riesgo de disfunción neuronal en esta zona del SNC asociada con memoria y capacidades cognitivas. 


\section{Conclusiones}

Con la mayor expectativa de vida y el envejecimiento de la población mundial, adquieren relevancia las investigaciones orientadas a tratar de establecer posibles factores genéticos, ambientales y patógenos que impactan en la salud humana. Los antecedentes expuestos en esta revisión contribuyen a aportar antecedentes que permitan entender y explicar la posible participación de HSV-1, agente viral de alta prevalencia en la población mundial, en desencadenar procesos inflamatorios crónicos que puedan generar disfunción neuronal $\mathrm{y}$ eventos neurodegenerativos progresivos. A pesar de que los riesgos que conlleva esta infección actualmente son subestimados, los estudios emergentes que asocian procesos de neurodegeneración con infección por HSV-1 avalan la conveniencia de realizar acciones preventivas tales como una mejor educación de la población acerca de las formas de minimizar el contagio y las ventajas de realizar un diagnóstico oportuno y terapias antivirales adecuadas.

Agradecimientos: Un especial agradecimiento al Dr. Heriberto Fernández por su colaboración en la edición de la presente revisión. El presente trabajo forma parte de la temática desarrollada con financiamiento FONDECYT 11080067 (CO). Las figuras del presente artículo fueron utilizadas previa autorización de Copyright (c) 2010 Elsevier B.V. y Copyright (c) 2000-2010 by John Wiley \& Sons, Inc.

\section{Referencias}

1. Mettenleiter. Pathogenesis of neurotropic herpesviruses: role of viral glycoproteins in neuroinvasion and transneuronal spread. Virus Res 2003; 92: 197-206.

2. Kieff E, Bachenheimer S, Roizman B. Size, composition, and structure of the deoxyribonucleic acid of herpes simplex virus subtypes 1 and 2. J Virol 1971; 8: 125-32.

3. Estevens J. Human herpesviruse: a consideration of the latent state. Microbiol Rev 1989; 53: 318-32.

4. Roizman B, Knipe M. Herpes simplex viruses and their replication. En: Fields Virology 4th ed. vol. 2. Knipe DM, Howley PM, Editores. Philadelphia Lippincott, Williams \& Wilkins; 2001.p. 2399-460.

5. Whitley RJ, Roizman B. Herpes simplex virus infections. Lancet 2001; 357:1513-8.

6. Xu F, Sternberg M, Kottiri B, McQuillan G, Lee F, Nah- mias A, et al. Trends in herpes simplex virus type 1 and type 2 seroprevalence in the United States. JAMA 2006; 296: 964-73.

7. Wagner E, Bloom D. Experimental investigation of Herpes simplex virus latency. Clin Microbiol Rev 1997; 10: 419-43.

8. Perkins D. Targeting apoptosis in neurological disease using the herpes simplex virus. J Cell Mol Med 2002; 3: 341-56.

9. Klapper P, Cleator, G, Longson M. Mild forms of herpes encephalitis. J Neurol Neurosurg Psych 1984; 47: 124750.

10. Schroth G, Gawehn J, Thron A, Voigt K. Early diagnosis of herpes simplex encephalitis by MRI. Neurology 1987; 37: 179-83.

11. Whitley RJ, Gnann JW. Viral Encephalitis: familiar infections and emerging pathogens. Lancet 2002; 359: 507-13.

12. Whitley RJ. Herpes simplex encephalitis: adolescents and adults. Antiviral Res 2006; 71: 141-8.

13. Kapur N, Barker S, Burrows E. Herpes simplex encephalitis: long term magnetic resonance imaging and neuropsychological profile. J Neurol Neurosurg Psych 1994; 57: 1334-42.

14. Damasio A, Van Hoesen G. The limbic system and the localization of herpes simplex encephalitis. J Neurol Neurosurg Psych 1985; 48: 297-301.

15. Esiri M. Herpes simplex encephalitis. An immunohistological study of the distribution of viral antigen within the brain. J Neurol Sci 1982; 54: 209-26.

16. Lee $\mathrm{S}$, Girard $\mathrm{S}$, Macina $\mathrm{D}$, Busà $\mathrm{M}$, Zafer $\mathrm{A}$, Belouchi A, et al. Susceptibility to mouse cytomegalovirus is associated with deletion of an activating natural killer cell receptor of the C-type lectin superfamily. Nat Genet 200?; 28: 42-45.

17. Thach D, Kimura T, Griffin D. Differences between C57BL/6 and BALB/cBy mice in mortality and virus replication after intranasal infection with neuroadapted Sindbis virus. J Virol 2000; 74: 6156-61.

18. Kastrukoff L, Kim S. Oligodendrocytes from human donors differ in resistance to herpes simplex virus 1 (HSV-1). Glia 2002; 38: 87-92.

19. Peinado A. Traveling slow waves of neural activity: a novel form of network activity in developing neocortex. J Neurosci 2000; 20: RC54.

20. Wozniak M, Shipley S, Combrinck M, Wilcock G, Itzhaki R. Productive herpes simplex virus in brain of elderly normal subjects and Alzheimer's disease patients. J Med Virol 2005; 75: 300-6.

21. Dobson C, Wozniak M, Itzhaki R. Do infectious agents play a role in dementia? Trends Microbiol 2003; 11:3127. 
22. Otth C, Zambrano A, Concha M. The possible link between herpes simplex virus type 1 infection and neurodegeneration. En: Current Hypotheses and Research Milestones in Alzheimer's disease. Maccioni RM y Perry G, Editores. New York- Heidelberg. SPRINGER-VERLAG; 2009. p. 181-8.

23. Ball M. Limbic predilection in Alzheimer dementia: is reactivated herpes virus involved? Can J Neurol Sci 1982; 9: 303-6.

24. Wilson L. Psychiatric aspects of acute viral encephalitis. En: Viruses, immunity, and mental disorders. Kurstak E, Lipowski ZJ, Morozov PV, Editores. Plenum Medical Book Company; 1987. p. 413-22.

25. Maccioni R, Muñoz J, Barbeito L. The molecular bases of Alzheimer's disease and other neurodegenerative disorders. Arch Med Res 2001; 32: 367-81.

26. Selkoe D. Alzheimer's disease: genes, proteins, and therapy. Physiol Rev 2001; 81: 741-66.

27. Goedert M, Spillantini M. A century of Alzheimer's disease. Science 2006; 314: 777-81.

28. Bottcher T, von Mering M, Ebert S, Meyding-Lamade U, Kuhnt U, Gerber J, et al. Differential regulation of Toll-like receptor mRNAs in experimental murine central nervous system infections. Neurosci Lett 2003; 344: 17-20.

29. Rock RB, Peterson PK. Microglia as a Pharmacological Target in Infectious and Inflammatory Diseases of the Brain. J Neuroimmune Pharmacol 2006; 1: 117-26.

30. Kurt-Jones EA, Chan M, Zhou S, Wang J, Reed G, Bronson $\mathrm{R}$, et al. Herpes simplex virus 1 interaction with Toll-like receptor 2 contributes to lethal encephalitis. Proc Natl Acad Sci 2004; 101: 1315-20.

31. Sato A, Linehan MM, Iwasaki A. Dual recognition of herpes simplex viruses by TLR2 and TLR9 in dendritic cells. Proc Natl Acad Sci 2006; 103 (46): 17343-8.

32. Aravalli RN, S Hu, JR Lokensgard. Inhibition of Tolllike Receptor Signaling in Primary Murine Microglia. J Neuroimmune Pharmacol 2008; 3: 5-11.

33. Dobson C, Itzhaki R. Herpes simplex virus type 1 and Alzheimer's disease. Neurobiol Aging 1999; 20: 457-65.

34. Itzhaki R, Lin W, Shang D, Wilcock G, Faragher B, Jamieson G. Herpes simplex virus type 1 in brain and risk of Alzheimer disease. Lancet 1997; 349: 241-4.

35. Leissring M, Sugarman M, La Ferla F. Herpes simplex virus infections and Alzheimer's disease. Drugs \& Aging 1998; 13: 193-8.

36. Denaro F, Staub P, Colmer J, Freed M. Coexistence of Alzheimer disease neuropathology with herpes simplex encephalitis. Cell Mol Biol 2003; 49: 1233-40.

37. Itzhaki R, Wozniak M, Appelt D, Balin B. Infiltration of the brain by pathogens causes Alzheimer's disease. Neurobiol Aging 2004; 25: 619-27.
38. Hill J, Gebhardt B, Azcuy A, Matthews K, Lukiw W, Steiner I, Thompson H, Ball M. Can a herpes simplex virus type 1 neuroinvasive score be correlated to their risk factors in Alzheimer's disease? Med Hypotheses 2005; 64: 320-7.

39. Itzhaki R, Dobson C, Shipley S, Wozniak M. The role of viruses and of APOE in dementia. Ann N Y Acad Sci 2004; 1019: 15-8.

40. Li, Y, Scott W, Hedges D, Zhang F, Gaskell P, Nance M, et al. Age at onset in two common neurodegenerative diseases is genetically controlled. Am J Hum Genet. 2002; 70: 985-93.

41. Chamelian L, Reis M, Feinstein A. Six-month recovery from mild to moderate traumatic brain injury: The role of apoe-epsilon4 allele. Brain 2004; 127: 2621-8.

42. Burgos JS, Ramírez C, Sastre I, et al. ApoE4 is more efficient than E3 in brain access by herpes simplex virus type 1. Gen Nerv Sys Dis 2003; 14: 1825-7.

43. Letenneur L, Pérès K, Fleury H, Garrigue I, BarbergerGateau P, Helmer C, Orgogozo J, et al. Seropositivity to herpes simplex virus antibodies and risk of Alzheimer's disease: a population-based cohort study. PLoS One 2008; 3: e3637.

44. Cribbs D, Azizeh B, Cotman C, La Ferla F. Fibril formation and neurotoxicity by a herpes simplex virus glycoprotein B fragment with homology to the Alzheimer's A peptide. Biochem 2000;39: 5988-94.

45. Wen Y, Yang S, Liu R, Simpkins J. Transient cerebral ischemia induces site-specific hyperphosphorylation of tau protein. Brain Res 2004; 1022: 30-8.

46. Planel E, Miyasaka T, Launey T, Chui D, Tanemura K, Sato S, Murayama O, Ishiguro K, Tatebayashi Y, Takashima A. Alterations in glucose metabolism induce hypothermia leading to tau hyperphosphorylation through differential inhibition of kinase and phosphatase activities: implications for Alzheimer's disease. J Neurosci 2004; 24: 2401-11.

47. De la Monte S, Neely T, Cannon J, Wands J. Oxidative stress and hypoxia-like injury cause Alzheimer-type molecular abnormalities in central nervous system neurons. Cell Mol Life Sci 2000; 57: 1471-81.

48. Guise S, Braguer D, Carles G, Delacourte A, Briand C. Hyperphosphorylation of tau is mediated by ERK activation during anticancer drug-induced apoptosis in neuroblastoma cells. J Neurosci Res 2001; 63: 257-67.

49. Stoothoff W, Johnson G. Hyperosmotic stress-induced apoptosis and tau phosphorylation in human neuroblastoma cells. J Neurosci Res 2001; 65: 573-82.

50. Lorio G, Ávila J, Díaz-Nido J. Modifications of tau protein during neuronal cell death. J Alzheimers Dis 2001; 3: 563-75. 
51. Zambrano A, Solís L, Salvadores N, Cortés M, Lerchundi R, Otth C. Neuronal cytoskeletal dynamic modification and neurodegeneration induced by infection with herpes simplex virus type 1. J Alzheimers Dis 2008; 14 : 259-69.

52. Otth C, Solís L, Villalba M, Lerchundi L, Martin C, Vio $\mathrm{K}$, et al. Neurodegeneration and Tau Hyperphosphorylation induced by Herpes Simplex Virus Type I infection on neurons and astrocytes. J Neurochem 2009; 110: 45.

53. Wozniak M, Frost A, Itzhaki R. Alzheimer's disease-specific tau phosphorylation is induced by herpes simplex virus type 1. J Alzheimer's Dis 2009;16: 341-50.
54. Lerchundi R, Neira R, Valdivia S, Vio K, Concha MI, Zambrano A, et al. Tau cleavage at D421 by caspase- 3 is induced in neurons and astrocytes infected with Herpes Simplex Virus type 1. J Alzheimer's Dis 2010; Nov 23.

55. Jamieson GA, Maitland NJ, Wilcock GK, Yates CM, Itzhaki RF. Herpes simplex virus type 1 DNA is present in specific regions of brain from aged people with and without senile dementia of the Alzheimer type. J Pathol 1992; 167: 365-8.

56. Jamieson GA, Maitland NJ, Wilcock GK, Craske J, Itzhaki RF. Latent herpes simplex virus type 1 in normal and Alzheimer's disease brains. J Med Virol 1991; 33: 224-7. 